\title{
Transmission Control Protocol Global Synchronization Problem in Wide Area Monitoring and Control Systems
}

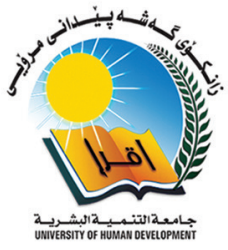

\author{
Yahya Ahmed Yahya ${ }^{1}$ and Ahmad T. Al-Hammouri ${ }^{2}$ \\ ${ }^{1}$ Department of Information Technology, Zakho Technical Institute, Duhok Polytechnic University Zakho, Duhok, \\ Kurdistan Region, Iraq, \\ ${ }^{2}$ Department of Network Engineering and Security, Jordan University of Science and Technology, Irbid, Jordan
}

\section{A B S T R A C T}

The electrical power network is a significant element of the critical infrastructure in modern society. Nowadays, wide area monitoring and control systems (WAMC) are becoming increasingly an important topic that motivates several researchers to improve, develop, and find the problems that hinder progress toward WAMC systems. WAMC is used to monitor and control the power network so the power network can be adapt to failures in automatic way. In this work, verification of the extent found a problem in transmission control protocol (TCP) which is called global synchronization and its impact on utilizing the buffer of the routers. A simulation models had been belt of WAMC system using OMNeT ++ to study the performance of TCP in two queuing algorithms for measuring transmission of phasor measurement units and to test if global synchronization problem occurs. Three scenarios were used to test the survival of this problem on the system. It is found that the problem of global synchronization occurred in two scenarios which in turn causes low utilization for a buffer of routers.

Index Terms: Global Synchronization, Phasor Measurement Units, Power Network, Transmission Control Protocol, Wide Area Monitoring and Control Systems

\section{INTRODUCTION}

Electric power grid is one of the most important topics in the modern societies. The sharp increase of demand on electricity, the electricity trade between the neighboring countries, and the long distances to transport electricity motivates the researchers and industries to propose and improve systems to monitor and control the electric power grid over a wide area. Hence, wide area monitoring and control systems (WAMC) became an important topic.

\begin{tabular}{|l|l|}
\hline \multicolumn{2}{|c|}{ Access this article online } \\
\hline DOI: 10.21928/uhdjst.v1n2y2017.pp7-12 & E-ISSN: 2521-4217 \\
& P-ISSN: 2521-4209 \\
\hline $\begin{array}{l}\text { Copyright @ } 2017 \text { Yahya and Al-Hammouri. This is an open access } \\
\text { article distributed under the Creative Commons Attribution Non- } \\
\text { Commercial No Derivatives License 4.0 (CC BY-NC-ND 4.0) }\end{array}$ \\
\hline
\end{tabular}

The communication network must transmit measurements with low latency and with high accuracy [1]. There are many factors affecting these two requirements, for example, the bandwidth, the type of medium, and the protocol that are used for transmission.

Phasor measurement unit (PMU) measurements can be transmitted over different types of transmission media such as wired or wireless. However, the best medium that can be used is the fiber-optic cable [1], [2]. The reason to choose fiber optic is the advantages including high data transfer rates, immunity to electromagnetic interference, and very large channel capacity [3].

There are many protocols used with WAMC system including the user datagram protocol (UDP), multiprotocol label switching (MPLS), resource reservation protocol (RSVP),

Corresponding author's e-mail: yahya.ahmed@dpu.edu.krd

Received: 10-03-2017

Accepted: 25-03-2017

Published: 29-08-2017 
and synchronous digital hierarchy. These protocols can be used individually or in combination (more than one of these protocols work with each other as one protocol), for example, using UDP with MPLS and RSVP as a one main protocol furnishes quality of services features.

Surveying the literature indicates that many protocols were used with WAMC systems except TCP. However, the NASPInet standard [4], [5] mentions that TCP can be used as the transport layer protocol to deliver PMU measurements. In general, architecture that is used in most WAMC systems is shown in Fig. 1.

The aim of this work is to study the effect of the global synchronization problem when we are using TCP with WAMC systems.

\section{TCP GLOBAL SYNCHRONIZATION PROBLEM}

TCP is one of more than a few transport protocols [6], [7]. It is reliable and stream oriented. In addition, TCP is connectionoriented, meaning it establishes a connection when data need to be transferred. The data are sent in packets and in an ordered manner at transport layer. It supports flow and congestion control [7].

Researchers after a deep study found a problem in TCP called global synchronization that can be defined as the pattern of each sender decreasing and increasing transmission rates at the same time as other senders [6], [7], [8].

As shown in Fig. 2, we built a topology to study the problem of global synchronization using $\mathrm{OMNeT}++$. The topology consists of the two senders, two receivers, and two routers. Each sender connected to one of two routers by bandwidth link equal to $100 \mathrm{Mbps}$ and propagation delay equal to $3 \mathrm{~ms}$. In the other hand, each receiver connects to the other router by bandwidth link equal to $100 \mathrm{Mbps}$ and propagation

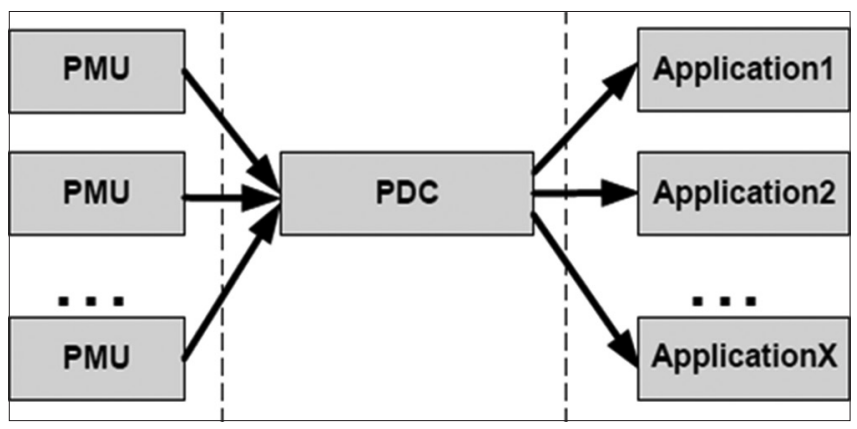

Fig. 1. General wide area monitoring and control Architecture [4] delay equal to $3 \mathrm{~ms}$. The two routers were connected by a bandwidth link equal to $10 \mathrm{Mbps}$ and propagation delay equal to $1 \mathrm{~ms}$. The buffer in the two routers was drop tail with 65-packet capacity.

After running the experiment, the result of congestion window (cwnd) is as shown in Fig. 3. The synchronization in both cwnd flows appeared clearly because both cwnds have to deal with the queue reaching its limits at the same time.

The low utilization of buffer seen can be clearer in Fig. 4 . The queue length is switching between full (65 packets) to nearly ( 5 packets). This oscillating load is caused by the synchronization of cwnd. This problem motivates the researchers, and they proposed many methods to solve this problem. Some researchers suggest adjusting router functionalities or modification in the transmission protocol parameters, and others suggested increasing the size of the router buffer capacity. However, these solutions will cause another unexpected problems because when increasing the size of router buffer, it is likely to increase queuing delays. The most adopted algorithm to reduce the global synchronization is random early drop queuing policy.

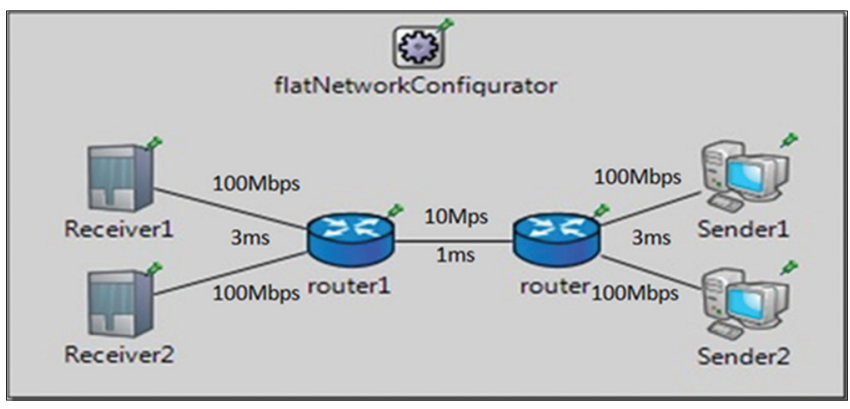

Fig. 2. Simple topology to test global synchronization

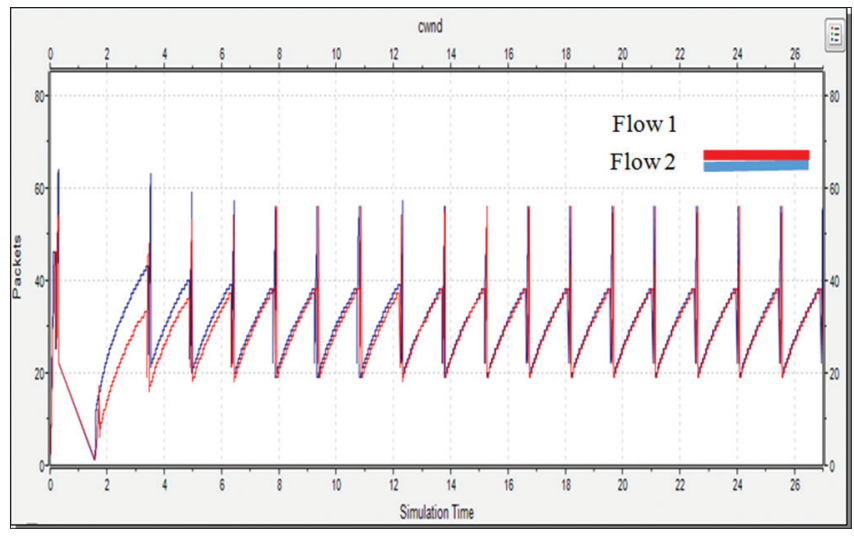

Fig. 3. Congestion windows of two senders 
It is liable in WAMC that several senders or PMUs can start sending in the same time. Hence, this study is to show how the global synchronization problem effects on WAMC.

\section{DESIGN OF A WAMC SYSTEM IN OMNET++}

\section{A. PMU Building}

This was to construct the first component of WAMC which is the PMU using $\mathrm{C}++$ language. The PMU generates packets in a certain period of time, and each packet contains three information's:

1. Sequence number of the packet: Which can be arranged systematically by giving a specific number to each packet.

2. Source ID: It is the number that helps to know from which PMU the packet had been generated.

3. Time stamp: It is the time of when the packet is generated until it reaches to PDC.

\section{B. PDC Level 1 (L1) Building}

This was to construct the second component of WAMC, which is the PDC using the $\mathrm{C}++$ language. The PDC is similar to the server of the computer, as its function is to collect all the measurements sent to it from the PMUs that are connected with the PDC.

The PDC function is to assemble all the packets that were produced from PMUs in one packet and to send it to PDC level 2.

For more details about PDC L1 flowchart, PDC L1 checks each packet after arriving from PMU "if this sequence number sent before that time," when "Yes" PDC will delete this packet, and if "No" PDC check whether "if this sequence number is a first time seen." When "Yes," PDC will add a time-out to this sequence number of the packet.

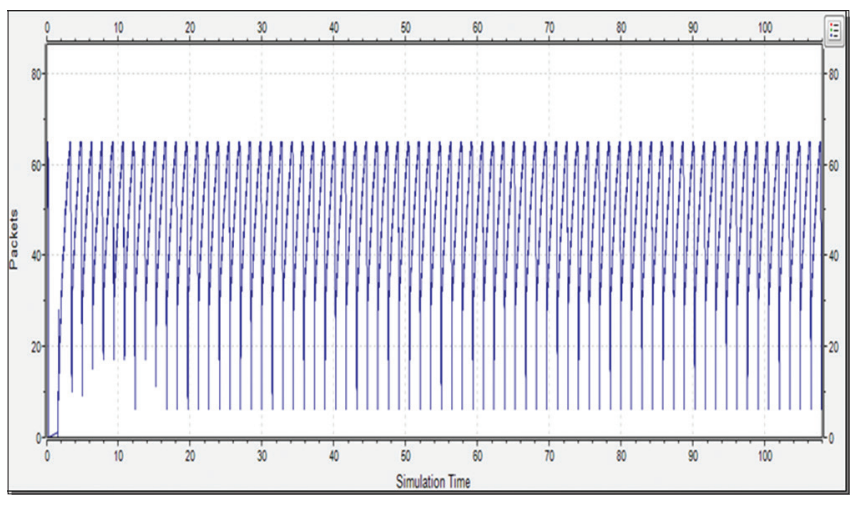

Fig. 4. The queue buffer of router
The benefit of this time-out is that when the timer fires PDC does not receive all packets from all PMUs, and then PDC will neglect and send only the arrived packets. After adding time-out to a packet with a sequence number, PDC will store the packet in the buffer and wait for packets that have the same sequence number. When the answer is "No," "if this sequence number is first time seen," PDC will store this packet in the buffer and checks whether that "all packets for one sequence number received or their time-out finish." When "No," wait until it is Yes.

When "Yes," PDC encapsulates the packets that have this sequence number in one packet that has a new sequence number, new source ID, and a new time stamp, then it will be sent to PDC L2.

\section{PDC Level 2 (L2) Building}

This was to construct the third component of WAMC, which is the PDC using the $\mathrm{C}++$ language. This PDC will receive the above mentioned single packet (produced in part II), then it will calculate the time of end-to-end latency and packet delivery ratio.

\section{SIMULATIONS AND RESULTS}

In this section, three scenarios were created as follows:

1. Twenty PMUs Scenario (high traffic rate)

2. Nineteen PMUs scenario (medium traffic rate)

3. Eighteen PMUs scenario (low traffic rate).

Through the study of the three scenarios, it was noticed in some cases of using the TCP protocol, the global synchronization problem occurred. Therefore, the study focuses on the buffer of the router, which may cause low utilization to the link bandwidth.

\section{A. Twenty PMUs Scenario (High Traffic Rate)}

The WAMC systems topology in this scenario has 20 PMUs, one PDC L1, and one PDC L2. Fig. 5 shows the topology of this scenario. There were two routers in the topology. The size of buffer capacity in each router is 100 packets. The links parameters that were used in all scenarios were listed in Table I. The packet size that sends from PMU is $512 \mathrm{~B}$, and the rate of date packet that generates from PMUs is $120 \mathrm{p} / \mathrm{s}$. A time-out of PDC is $0.024 \mathrm{~s}$. Table I shows all scenarios parameters.

The buffer result of router when using TCP with the FIFO queue algorithm is shown in Fig. 6. 
Yahya Ahmed Yahya and Ahmad T. Al-Hammouri: TCP Global Synchronization Problem in WAMC Systems

\begin{tabular}{lcc}
\multicolumn{2}{c}{$\begin{array}{c}\text { TABLE I } \\
\text { Links Parameters of All Scenarios }\end{array}$} \\
\hline Link between & Data rate (bps) & $\begin{array}{c}\text { Propagation } \\
\text { delay (ms) }\end{array}$ \\
\hline PMUs and router & $150 \mathrm{M}$ & $3-6$ \\
Router and PDC L1 & $10 \mathrm{M}$ & 1 \\
PDC L1 and router1 & $150 \mathrm{M}$ & 3 \\
Router1 and PDC L2 & $150 \mathrm{M}$ & 3 \\
\hline
\end{tabular}

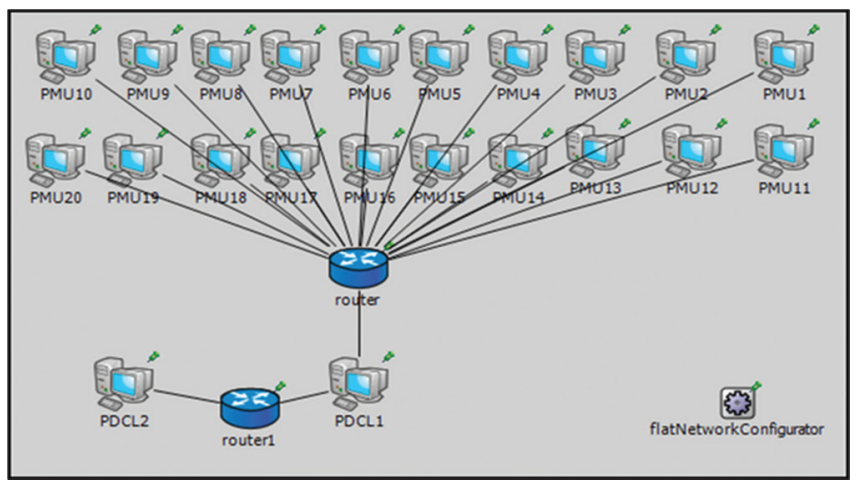

Fig. 5. The topology in 20 phasor measurement units scenario

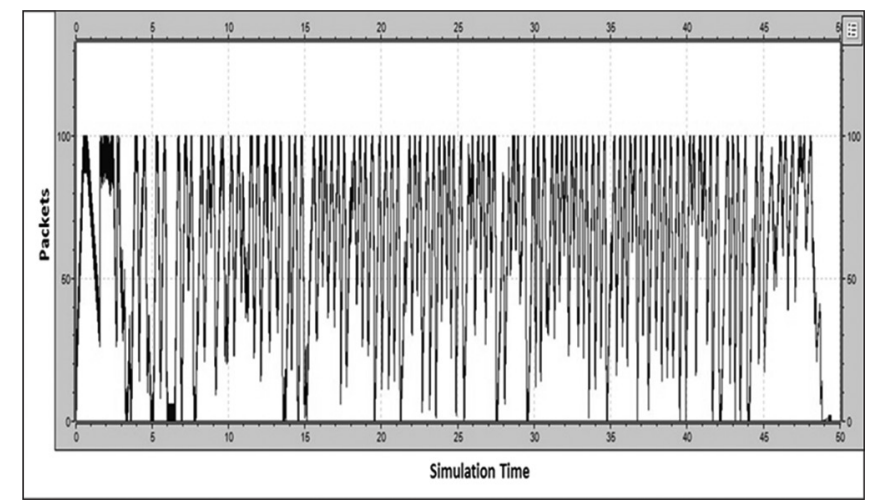

Fig. 6. The buffer of router when using transmission control protocol with the FIFO queue algorithm

The buffer exhibits oscillation between 0 and 100, which means that global synchronization had occurred in this scenario. When we used TCP with RED queue algorithm, the results are shown in Fig. 7.

Now, the buffer exhibits oscillation, but this oscillation did not to exceed 50 packets as before using TCP with FIFO.

\section{B. Nineteen PMUs Scenario (Medium Traffic Rate)}

The parameter of this scenario is as mentioned in 20 PMUs scenario. The only difference between the scenarios is the number of PMUs. Fig. 8 shows topology of 19 PMUs scenario.

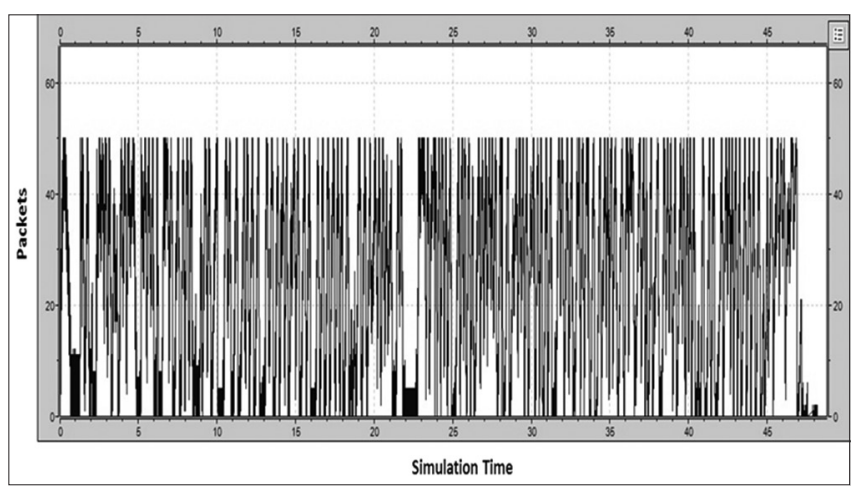

Fig. 7. The buffer of router when using transmission control protocol with the RED queue algorithm

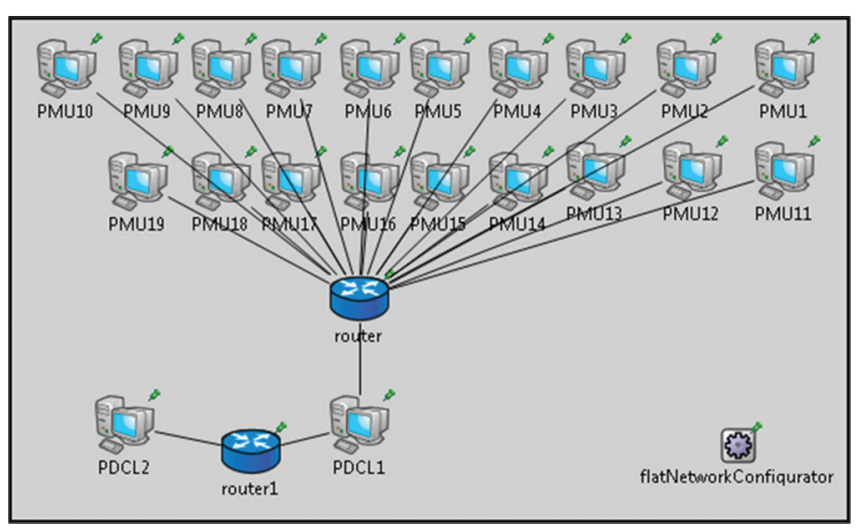

Fig. 8. The topology in 19 phasor measurement units scenario

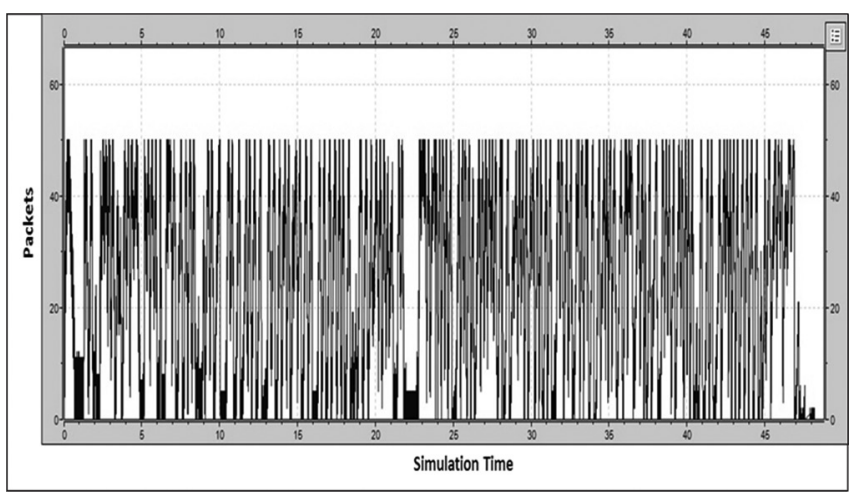

Fig. 9. The buffer of router when using transmission control protocol with the FIFO queue algorithm

For 19 PMUs scenario (TCP protocol and FIFO), the result of buffer is shown in Fig. 9.

The buffer exhibits oscillation between 0 and 100, which means that global synchronization had occurred in this scenario. However, the exhibits oscillation is lower than the scenario of 20 PMUs. When we used TCP with RED, the results are shown in Fig. 10. 


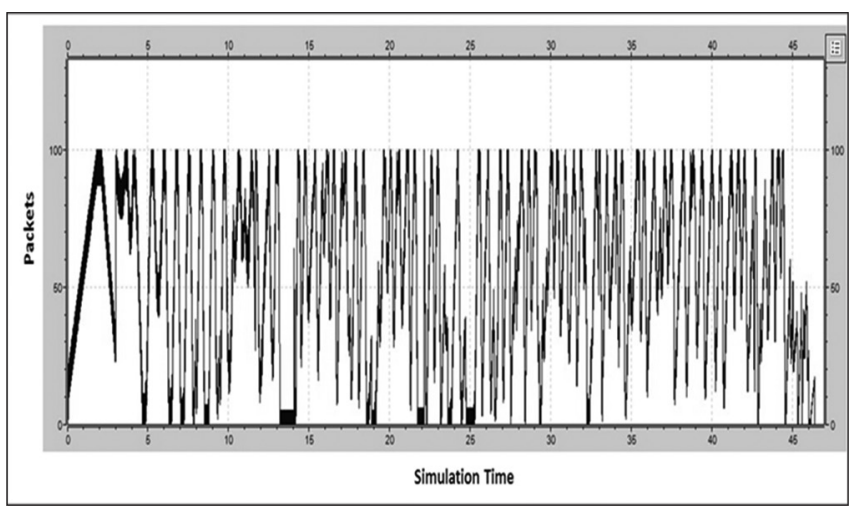

Fig. 10. The buffer of router when using transmission control protocol with the RED queue algorithm

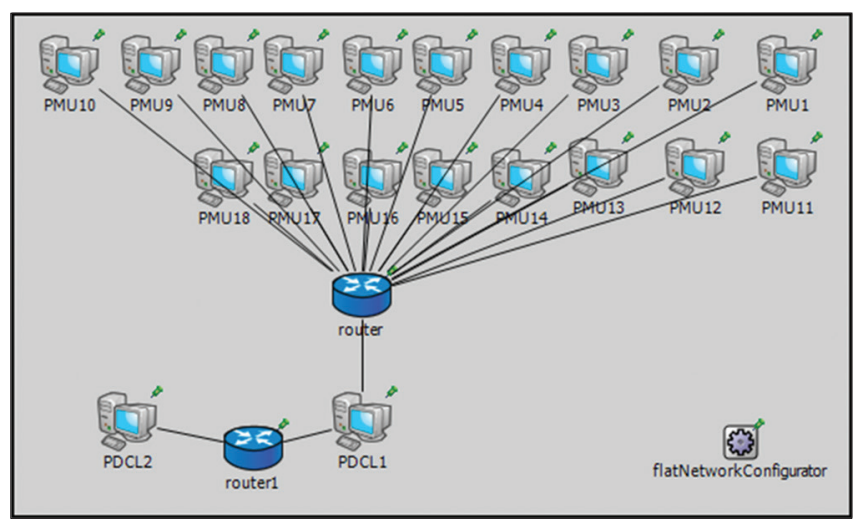

Fig. 11. The topology in 18 phasor measurement units scenario

The buffer exhibits oscillation, but this oscillation did not exceed 50 packets as before using TCP with FIFO. The oscillation in this scenario is the same of scenario 20 PMUs.

\section{Eighteen PMUs Scenario (Low Traffic Rate)}

The parameter of this scenario is mentioned in 20 and 19 PMUs scenario. The only difference between the scenarios is the number of PMUs. Fig. 11 shows topology of 18 PMUs scenario.

In 18 PMUs scenario, there is no oscillation of packets which means that there is no global synchronization in this scenario. The buffer exhibits oscillation between 0 to 10 or 11 while the buffer size is 100 packets. Figs. 12 and 13 show the buffer of routers when we used TCP with FIFO and RED queue algorithm of router was used.

\section{CONCLUSION}

In this study, WAMC system using OMNeT++ was created and applied TCP protocol (FIFO and RED) queue algorithm

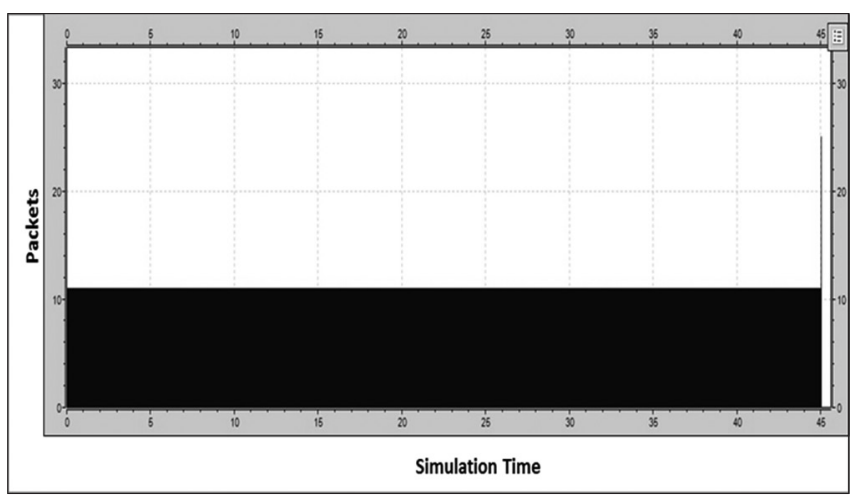

Fig. 12. The buffer of router when we used transmission control protocol with the FIFO queue algorithm.

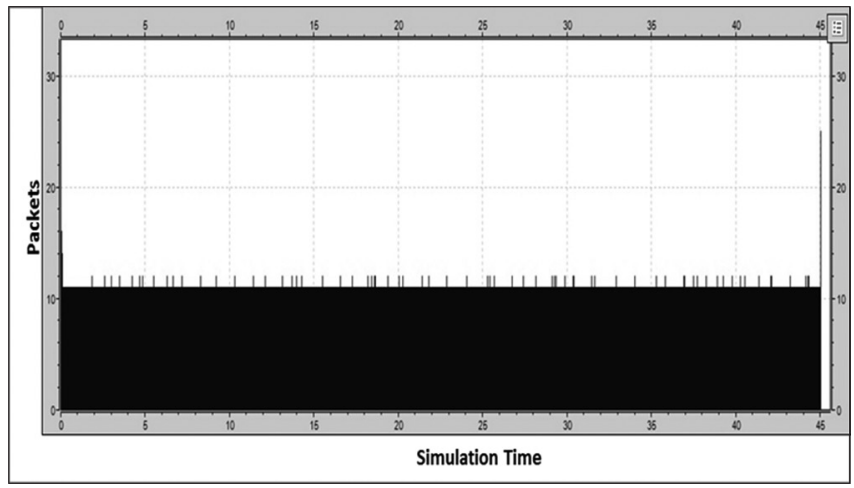

Fig. 13. The buffer of router when we used transmission control protocol with the RED queue algorithm

that was used to deliver the measurements and control information over the networks.

The global synchronization problem occurred with the TCP protocol when the PMUs sent the measurements in the synchrony manner in two scenarios (20 and 19 PMUs) in other words when the traffic rate is high and medium. Whereas in the scenario of 18 PMUs when traffic rate is low, the global synchronization was not occurred. According to this study, it is recommended not to used TCP protocol in WAMC systems to do not use TCP protocol in WAMC system during have high rate traffic.

\section{REFERENCES}

[1] C. Moustafa and L. Nordström. "Investigation of communication delays and data incompleteness in multi-pmu wide area monitoring and control systems." Electric Power and Energy Conversion Systems, EPECS'09. International Conference on IEEE, 2009.

[2] H. Erich, H. Khurana and T. Yardley. "Exploring convergence for SCADA networks." Innovative Smart Grid Technologies (ISGT), 
IEEE PES, IEEE, 2011.

[3] C. Moustafa, K. Zhu and L. Nordstrom. Survey on Priorities and Communication Requirements for PMU-Based Applications in the Nordic Region. PowerTech, 2009.

[4] C. Moustafa, A. Layd and L. Jordan. "PMU traffic shaping in IP. based wide area communication." Critical Infrastructure (CRIS), $20105^{\text {th }}$ International Conference on IEEE, 2010.

[5] Y. Yorozu, M. Hirano, K. Oka and Y. Tagawa. "Electron spectroscopy studies on magneto-optical media and plastic substrate interface," IEEE Translation Journal on Magnetics in Japan, vol. 2, pp. 740-741,
Aug. 1987. (Digests $9^{\text {th }}$ Annual Conf. Magnetics Japan, p. 301, 1982).

[6] Z. Lixia and C. David. "Oscillating behavior of network traffic: A case study simulation". Internetworking: Research and Experience, vol. 1, pp. 101-112, 1990.

[7] S. Chakchai. "Loss synchronization of TCP connections at a shared bottleneck link." Department of Computer Science and Engineering, St. Louis: Washington University, 2006.

[8] H. Sofiane and R. David "loss synchronization, router buffer sizing and high-speed TCP versions: Adding RED to the mix". IEEE $34^{\text {th }}$ Conference on Local Computer Networks, 2009. 